\title{
Concreto leve estrutural: influência da argila expandida na microestrutura da zona de transição pasta/agregado
}

Structural lightweight concrete: influence of expanded clay on the microstructure of the interfacial transition zone

\section{João Adriano Rossignolo}

\section{Resumo}

$\mathbf{E}$ ste trabalho apresenta os resultados de uma pesquisa sobre o efeito do agregado leve brasileiro (argila expandida) na zona de transição pasta/agregado em concretos leves estruturais. Utilizou-se um microscópio eletrônico de varredura (MEV) associado a um sistema de análise quantitativa (espectrografia de raios X por dispersão de energias - EDS) para a avaliação da espessura da zona de transição. No concreto de referência, com brita basáltica, a zona de transição apresentou espessura de $55 \mu \mathrm{m}$, enquanto nos concretos com argila expandida observou-se a redução desse valor para $30 \mu \mathrm{m}$. Verificou-se, ainda, que a zona de transição dos concretos com argila expandida apresentou menores teores de poros e de hidróxido de cálcio do que o observado nos concretos com brita basáltica.

Palavras-chave: Concreto leve estrutural. Argila expandida. Zona de transição. MEV. EDS.

João Adriano Rossignolo Departamento de Arquitetura e
Urbanismo, Escola de

Engenharia de São Carlos

Universidade de São Paulo

Av. Trabalhador São-Carlense,

São Carlos - SP - Brasil CEP 13570-960

E-mail: jarossig@sc.usp.br

Recebido em 27/05/09 Aceito em 17/11/09

\section{Abstract}

This paper presents the results of a study on the effect of expanded clay on the microstructure of the interfacial transition zone (ITZ) between Portland cement paste and aggregates. A scanning electron microscope (SEM) equipped with an energy dispersive $X$-ray analysis system (EDX) was used to determine the ITZ thickness. In plain concrete a marked ITZ around the aggregate particles (55 was observed, while in concretes with expanded clay, the ITZ was less pronounced (30 $\mathrm{mm}$ ). It was also verified that the ITZ of concrete with expanded clay had lower levels of porosity and calcium hydroxide than that observed in plain concrete.

Keywords: Experiment Structural lightweight concrete. Expanded clay. Interfacial transition zone. SEM. EDX. 


\section{Introdução}

Segundo Monteiro (1993) e Paulon (2005), existe uma forte relação entre a espessura e a qualidade da zona de transição e as propriedades mecânicas e a durabilidade dos concretos, o que indica a grande importância do estudo da zona de transição visando à melhoria do desempenho do concreto.

Alguns estudos sobre a microestrutura de concretos com agregados leves demonstraram que a interação entre esse tipo de agregado e a pasta de cimento é diferente da ocorrida nos concretos com agregados convencionais (CHEN; SCHNEIDER, 1998; ROSSIGNOLO, 2003; VIEIRA, 2000; WASSERMAN; BENTUR, 1996, 1997, 1998).

A natureza da interação entre os agregados leves e a pasta de cimento depende essencialmente do teor de umidade e da porosidade permeável da região externa do agregado. Quando os agregados leves utilizados forem previamente saturados ou apresentarem uma camada externa com baixa porosidade permeável, a natureza da microestrutura da zona de transição será muito próxima da observada nos concretos com agregados convencionais.

Entretanto, para agregados leves com baixo teor de umidade e com porosidade permeável na face externa, como a argila expandida brasileira, ocorre a redução da espessura da zona de transição assim como a quantidade de poros e de hidróxido de cálcio $(\mathrm{CH})$, em função da diminuição da relação água/cimento da pasta nessa região, ocasionada pela absorção de água do agregado. Esse efeito é denominado de "filtragem" ou "densificação" (LO; GAO; JEARY, 1999; SARKAR; CHANDRA; BERNTSSON, 1992; VIEIRA, 2000; ZHANG; GJ $\phi R V$, 1990; ZHANG; GJ $\phi R V$, 1992).

Segundo Zhang e Gj申Rv (1990), a interface entre o agregado leve e a pasta de cimento apresenta baixa porosidade e caracteriza-se pela alta ancoragem mecânica entre o agregado leve e a pasta de cimento, conseqüência da absorção de água e da rugosidade da superfície do agregado.
Holm e Bremner (2000) examinaram estruturas de concreto com agregados leves expostas em ambientes marinhos há mais de 60 anos e verificaram que o agregado leve apresentava boa aderência à pasta e que a quantidade de sílica presente na interface pasta/agregado era maior do que a presente na pasta de cimento.

Os estudos realizados por Wasserman e Bentur (1996) e Elsharif, Cohen e Olek (2005) indicaram que a redução da espessura da zona de transição pasta/agregado melhora o desempenho das propriedades relacionadas à resistência mecânica e à durabilidade dos concretos leves.

Com isso, este trabalho dedica-se ao melhor entendimento da influência da argila expandida brasileira na microestrutura da zona de transição pasta/agregado em concretos leves estruturais, por meio da análise comparativa com concretos com brita basáltica. Utilizou-se a análise quantitativa por espectrografia de raios $\mathrm{X}$ por dispersão de energia (EDS) associada ao microscópio eletrônico de varredura (MEV) para a análise da zona de transição pasta/agregado.

\section{Materiais e métodos}

Os materiais utilizados para a produção dos concretos analisados nesta pesquisa foram: cimento Portland de alta resistência inicial (CPV ARI), com massa específica de $3,12 \mathrm{~g} / \mathrm{cm}^{3}$ e área específica Blaine de $4.687 \mathrm{~cm}^{2} / \mathrm{g}$; areia natural quartzosa com dimensão máxima de 2,4 mm; argila expandida de fabricação nacional, com dimensão máxima característica de 9,5 mm, fornecida pela empresa CINEXPAN Argila Expandida Ltda.; e brita basáltica com dimensão máxima característica de 9,5 mm (Tabela 1). A Tabela 2 apresenta o resultado da análise química da argila expandida CINEXPAN 1506.

\begin{tabular}{|c|c|c|c|c|c|c|}
\hline \multirow{2}{*}{ Agregado } & \multirow{2}{*}{$\begin{array}{c}\text { Dimensão máxima } \\
\text { característica }\left(\mathrm{D}_{\max }\right) \\
\text { NBR 7211(2009) } \\
(\mathbf{m m}) \\
\end{array}$} & \multirow{2}{*}{$\begin{array}{c}\text { Massa específica } \\
\text { NBRNM } 52(2003) \\
\left(\mathbf{g} / \mathrm{cm}^{3}\right)\end{array}$} & \multirow{2}{*}{$\begin{array}{c}\text { Massa unitária } \\
\text { NBRNM 45 (2006) } \\
\left(\mathrm{g} / \mathrm{cm}^{3}\right)\end{array}$} & \multicolumn{3}{|c|}{$\begin{array}{c}\text { Absorção de água (\%) } \\
\text { (em massa)* }\end{array}$} \\
\hline & & & & $30 \mathrm{~min}$ & $60 \mathrm{~min}$ & $24 \mathrm{~h}$ \\
\hline Areia quartzosa & 2,4 & 2,63 & 1,49 & - & - & - \\
\hline Brita basáltica & 9,5 & 2,87 & 1,32 & - & - & - \\
\hline CINEXPAN 1506 & 9,5 & 1,11 & 0,59 & 2,7 & 3,5 & 7,0 \\
\hline
\end{tabular}

* Método de ensaio proposto por Tezuka (1973)

Tabela 1 - Características e propriedades dos agregados 


\begin{tabular}{cc}
\hline Composto & \% \\
\hline $\mathrm{SiO}_{2}$ & 62,3 \\
\hline $\mathrm{Al}_{2} \mathrm{O}_{3}$ & 17,7 \\
\hline $\mathrm{Fe}_{2} \mathrm{O}_{3}$ & 10,3 \\
\hline $\mathrm{MgO}$ & 2,8 \\
\hline $\mathrm{K}_{2} \mathrm{O}$ & 4,1 \\
\hline $\mathrm{TiO}_{2}$ & 1,0 \\
\hline $\mathrm{Na}_{2} \mathrm{O}$ & 0,3 \\
\hline $\mathrm{CaO}$ & 0,4 \\
\hline $\mathrm{PF}$ & 0,7
\end{tabular}

Tabela 2 - Análise química da argila expandida CINEXPAN 1506

Foram produzidos dois tipos de concreto, com argila expandida e com brita basáltica, com relação água/cimento de 0,40 , utilizando-se a relação cimento/agregado de 1:1, em massa, com a composição dos agregados de $30 \%$ de areia e $70 \%$ de argila expandida ou de brita basáltica, em massa.

Foram moldados cinco corpos-de-prova de $35 \mathrm{~mm}$ de diâmetro e $70 \mathrm{~mm}$ de altura para cada tipo de concreto. Após a moldagem, os corpos-de-prova foram acondicionados em câmara úmida (23 \pm 2 ${ }^{\circ} \mathrm{C}$ e $95 \pm 5 \%$ UR) e desmoldados após 24 horas. Em seguida, os corpos-de-prova permaneceram na câmara úmida por mais 27 dias. Após o período de cura úmida, os corpos-de-prova foram envolvidos em um filme plástico e armazenados em uma sala climatizada $\left(25 \pm 3{ }^{\circ} \mathrm{C}\right.$ e $60 \pm 15 \%$ UR), até a realização das análises experimentais, que ocorrem aos 100 dias de idade das amostras.

$\mathrm{Na}$ escolha e na análise das amostras de concreto, para o estudo da interface entre o agregado e a pasta de cimento, foram tomados os seguintes cuidados: foram utilizadas as amostras do centro do corpo-de-prova; fez-se o corte das amostras em planos perpendiculares ao sentido de moldagem; e foram analisadas as interfaces ao redor dos agregados que apresentaram dimensão máxima $(9,5 \mathrm{~mm})$ na superfície analisada, evitando, assim, regiões com probabilidade de exsudação, como as partes superior e inferior do agregado, considerando o sentido da moldagem.

$\mathrm{Na}$ preparação para análise no $\mathrm{MEV}$ (modo elétrons retroespalhados), as amostras de concreto foram cortadas em dimensões de $3,0 \mathrm{~cm}$ x $3,0 \mathrm{~cm}$, com $1 \mathrm{~cm}$ de espessura, utilizando serra de baixa rotação, com disco diamantado, lubrificado com água. Após o corte, as amostras foram imersas em álcool isopropílico durante 24 horas para interrupção da hidratação da matriz de cimento. Em seguida, as amostras foram submetidas ao processo de secagem, permanecendo 12 horas em estufa ventilada, com temperatura de $35^{\circ} \mathrm{C}$ e 24 horas em dissecador à temperatura de $23 \pm 2{ }^{\circ} \mathrm{C}$. Ao fim dessa etapa, as amostras foram submetidas ao processo de impregnação a vácuo, com embutimento a frio, utilizando-se uma resina epóxi com baixa viscosidade, baixo índice de refração e baixa retração.

Inicialmente, realizou-se o polimento das amostras utilizando-se lixas de carbeto de silício 300, 400, 600 e 1.000 em politriz de disco rotativo com lubrificação de óleo mineral. As amostras foram limpas, utilizando-se ultra-som com resfriamento em álcool isopropílico, após a finalização do polimento em cada tipo de lixa. Fez-se o polimento final utilizando-se pasta de diamante (suspensão de diamante em óleo mineral) com três diferentes tamanhos dos grãos: $6 \mu \mathrm{m}, 1 \mu \mathrm{m}$ e $0,25 \mu \mathrm{m}$. Após o polimento final, as amostras foram novamente submetidas ao processo de limpeza em ultra-som, com resfriamento em álcool isopropílico. Após o polimento, as amostras receberam uma camada condutora de carbono e foram mantidas em dissecador à temperatura ambiente, até o momento da análise.

A análise da zona de transição entre o agregado e a pasta de cimento para os dois tipos de concreto foi feita por meio da verificação semiquantitativa dos principais produtos da hidratação de cimento nas proximidades do agregado, tais como $\mathrm{CH}$ (hidróxido de cálcio), C-S-H ${ }^{1}$ (silicato de cálcio hidratado), AFt (etringita) e AFm (monosulfoaluminato de cálcio).

Essa análise foi feita utilizando-se os resultados das pesquisas realizadas por Taylor e Newbury (1984), Wasserman e Bentur (1996) e Kjellsen, Wallevik e Fjällberg (1998), em que foram apresentados valores para as relações das massas

\footnotetext{
${ }^{1}$ Os químicos de cimento normalmente utilizam as seguintes
} abreviações: $\mathrm{C}=\mathrm{CaO} ; \mathrm{S}=\mathrm{SiO}_{2} ; \mathrm{H}=\mathrm{H}_{2} \mathrm{O}$. 
atômicas de alguns óxidos presentes na pasta de cimento ( $\mathrm{SiO} 2$ [Si], $\mathrm{CaO}$ [Ca], $\mathrm{Fe} 2 \mathrm{O} 3$ [Fe], $\mathrm{Al} 2 \mathrm{O} 3$ [Al] e SO3 [SO3]), para associá-las à presença dos produtos do cimento hidratado, como apresentado abaixo.

\section{C-S-H:}

\section{$0,8 \leq \mathrm{Ca} / \mathrm{Si} \leq 2,5 \quad(\mathrm{Al}+\mathrm{Fe}) / \mathrm{Ca} \leq 0,2$}

CH:

$\mathrm{Ca} / \mathrm{Si} \geq 10 \quad(\mathrm{Al}+\mathrm{Fe}) / \mathrm{Ca} \leq 0,04 \quad \mathrm{SO} 3 / \mathrm{Ca} \leq 0,04$ AFt e AFm:

$\mathrm{Ca} / \mathrm{Si} \geq 4 \quad(\mathrm{Al}+\mathrm{Fe}) / \mathrm{Ca}>0,4 \quad \mathrm{SO} 3 / \mathrm{Ca}>0,15$

Assim, a determinação da espessura da zona de transição pasta/agregado para os dois tipos de concreto, com 100 dias de idade, foi realizada com a representação dos perfis de $\mathrm{Ca} / \mathrm{Si},(\mathrm{Al}+\mathrm{Fe}) / \mathrm{Ca}$ e $\mathrm{SO}_{3} / \mathrm{Ca}$, utilizando-se a análise semiquantitativa por espectrografia de raios $\mathrm{X}$ por dispersão de energia (EDS) associada ao microscópio eletrônico de varredura (MEV), em vários pontos ao longo de uma linha perpendicular à interface pasta/agregado. A análise das amostras foi realizada no Núcleo de Química (NQ) do Departamento de Materiais de Construção (DMC) do Laboratório Nacional de Engenharia Civil (LNEC), em Lisboa, Portugal, utilizando-se um microscópio eletrônico da marca JEOL, modelo JSM-6400, com filamento de tungstênio.

Para cada tipo de concreto foram analisados 10 perfis, com 15 pontos cada, ao longo de uma linha perpendicular à interface pasta/agregado. Foram utilizadas cinco amostras polidas para cada tipo de concreto, sendo analisados dois perfis em cada amostra. O comprimento do perfil analisado, perpendicular à interface, foi de aproximadamente $180 \mu \mathrm{m}$, sendo $40 \mu \mathrm{m}$ no agregado e $140 \mu \mathrm{m}$ na pasta de cimento. As Figuras 1 e 2 apresentam exemplos do perfil analisado nas amostras polidas de concreto.

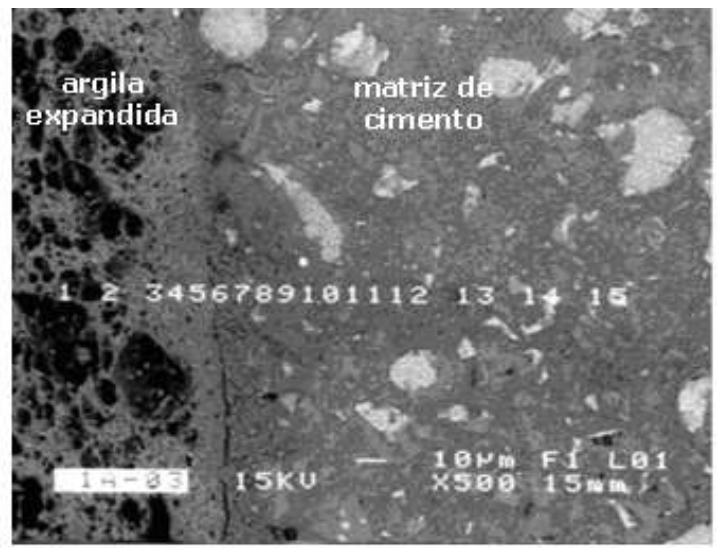

Figura 1 - Micrografia (MEV - modo elétrons retroespalhados) do perfil de análise nas amostras polidas de concreto com argila expandida

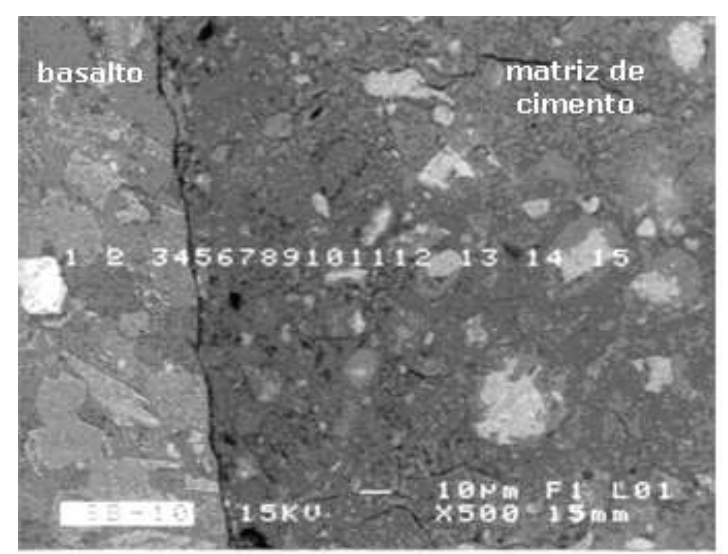

Figura 2 - Micrografia (MEV - modo elétrons retroespalhados) do perfil de análise nas amostras polidas de concreto com agregado basáltico 
Além das análises dos perfis ao longo de uma linha perpendicular à interface pasta/agregado utilizando-se EDS associado ao MEV (modo elétrons retroespalhados), também foram realizadas análises de imagens obtidas no MEV (modo elétrons retroespalhados) das amostras polidas para verificação dos teores de poros e grãos não hidratados de cimento Portland na região da zona de transição entre a pasta e o agregado. Para a análise de imagens selecionaram-se 10 faixas paralelas à linha da zona de transição pasta/agregado com $10 \mu \mathrm{m}$ de espessura e $150 \mu \mathrm{m}$ de largura (Figura 3). A contagem dos pixels pretos e brancos fornece as informações sobre a quantidade de poros e grãos não hidratados de cimento, respectivamente, em relação à área total da imagem, conforme procedimento descrito em Elsharif, Cohen e Olek (2003).

\section{Apresentação e análise dos resultados}

As Figuras 4 e 5 apresentam os resultados das análises dos perfis ao longo de uma linha perpendicular à interface pasta/agregado utilizando-se EDS associado ao MEV. Os valores obtidos para as relações $(\mathrm{Al}+\mathrm{Fe}) / \mathrm{Ca}$ e $\mathrm{SO}_{3} / \mathrm{Ca}$ indicam que nenhum dos concretos estudados apresentou teores significativos de AFm ou de $\mathrm{AFt}$, assumindo-se que os elementos $\mathrm{Al}, \mathrm{Fe}$ e $\mathrm{SO}_{3}$ estão presentes na pasta de cimento predominantemente nas fases $\mathrm{AFm}$ e $\mathrm{AFt}$ Observa-se, também, que não houve uma região preferencial para a formação de AFm e AFt, como, por exemplo, na zona de transição pasta/agregado.

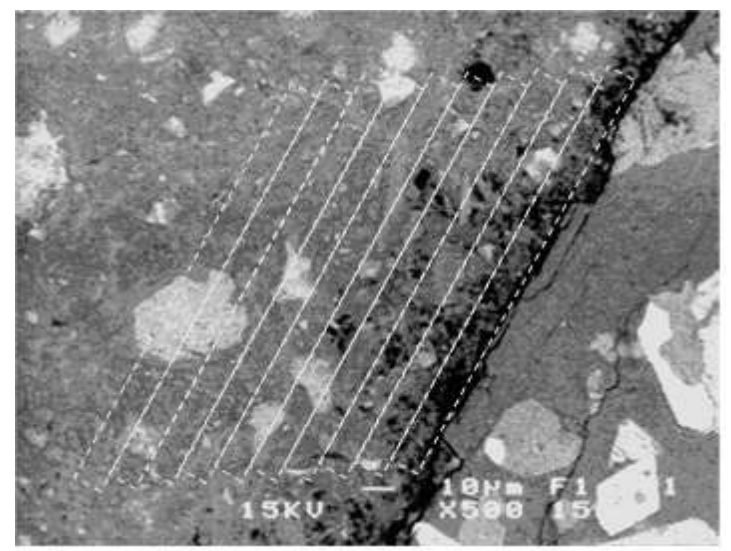

Figura 3 - Micrografia (MEV - modo elétrons retroespalhados) com as faixas delimitadas para análise de imagens dos poros e dos grãos de cimentos não hidratados (concreto com brita basáltica)

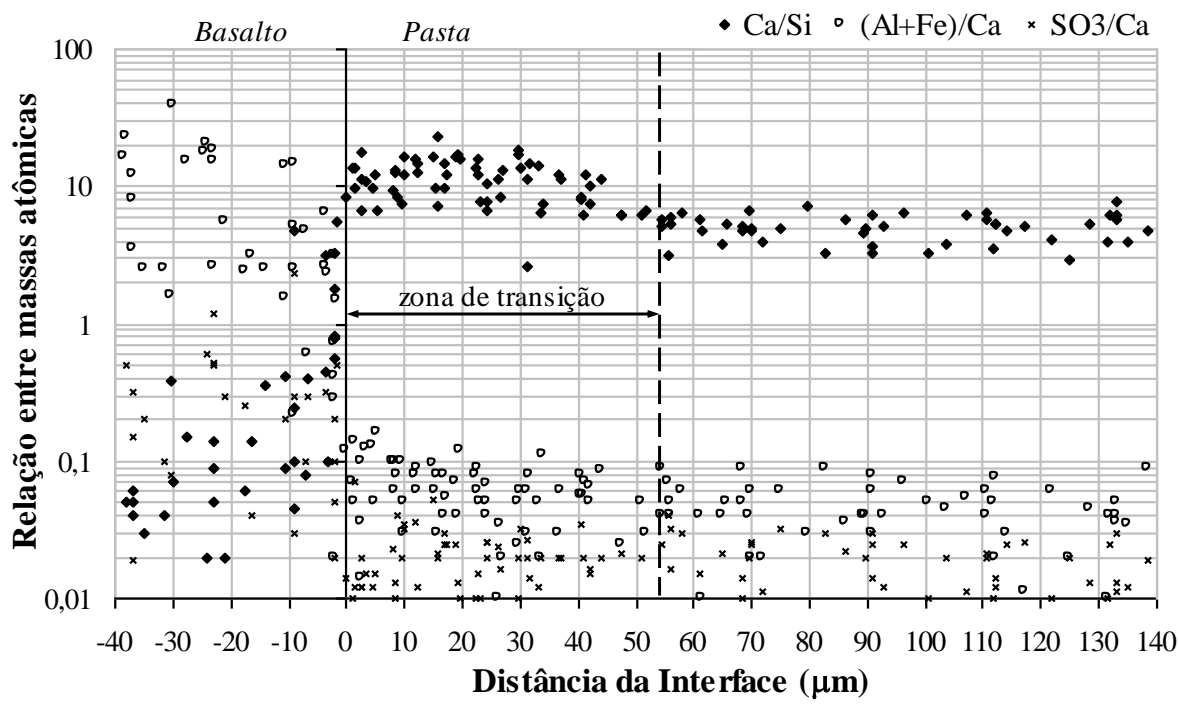

Figura 4 - Relações entre Ca/Si para o concreto com agregado basáltico 


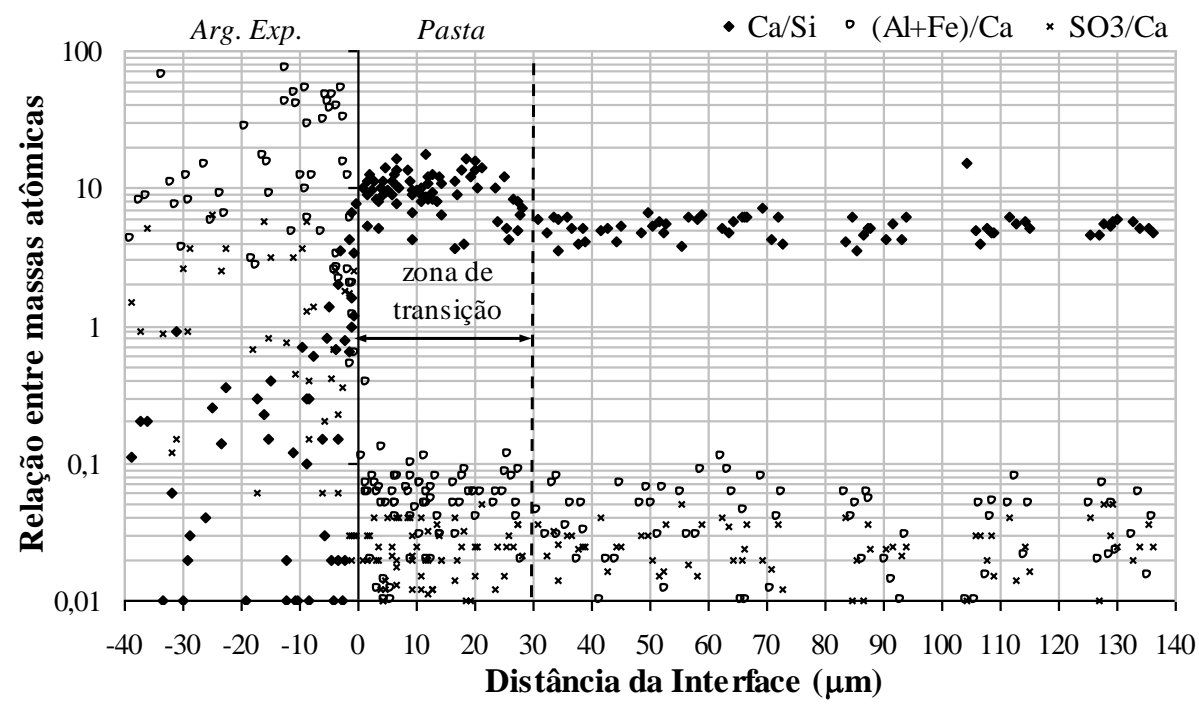

Figura 5 - Relações entre $\mathrm{Ca} / \mathrm{Si}$ para o concreto com argila expandida

Com isso, a espessura da zona de transição foi determinada por meio da análise do comportamento da relação $\mathrm{Ca} / \mathrm{Si}$, que apresentou variação significativa na região próxima ao agregado, possibilitando, assim, a identificação da zona de transição. A espessura da zona de transição foi definida como a distância entre a interface pasta/agregado e o ponto em que os valores da relação $\mathrm{Ca} / \mathrm{Si}$ apresentaram estabilização.

Nos concretos com argila expandida, o valor obtido para a espessura da zona de transição foi de $30 \mu \mathrm{m}$, enquanto nos concretos com agregado basáltico foi de $55 \mu \mathrm{m}$, demonstrando, assim, que a absorção de água da argila expandida promove a redução da espessura da zona de transição, em função da redução da relação água/cimento da pasta nessa região.

Esses resultados são corroborados pelos dados obtidos na análise do teor de poros e de grãos não hidratados de cimento Portland, apresentados nas Figuras 6 e 7, respectivamente. Nesses casos, a espessura da zona de transição pode ser definida como a distância entre a interface pasta/agregado e o ponto em que os valores dos teores de porosidade ou de grãos não hidratados de cimento Portland apresentaram estabilização em torno dos valores obtidos para a pasta de cimento. Os resultados dessas análises indicam que as espessuras da zona de transição dos concretos com argila expandida e com brita basáltica foram de, aproximadamente, $30 \mu \mathrm{m} \quad$ e $55 \quad \mu \mathrm{m}$ respectivamente.

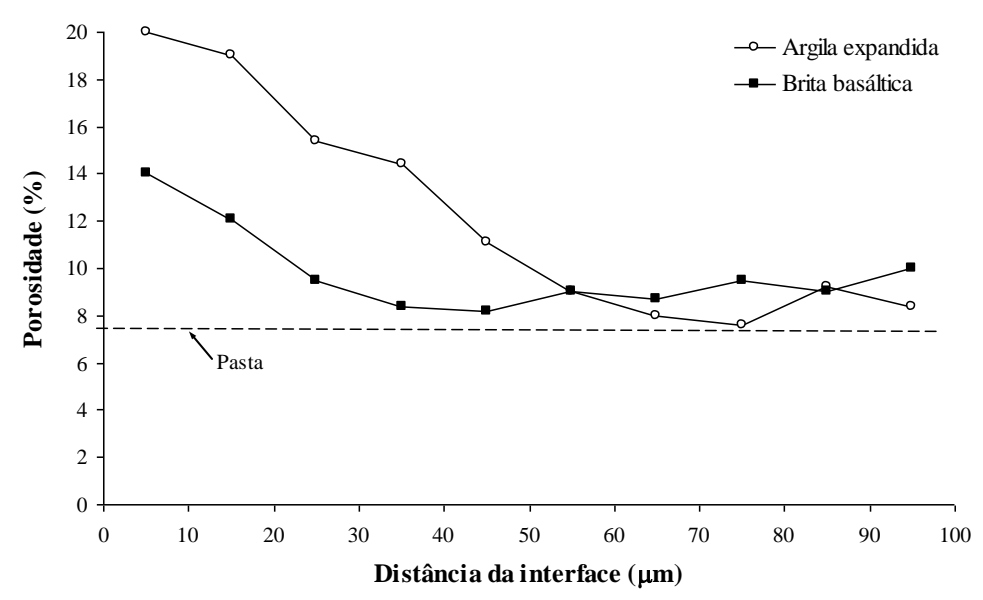

Figura 6 - Valores da porosidade da pasta em função da distância da interface pasta/agregado 


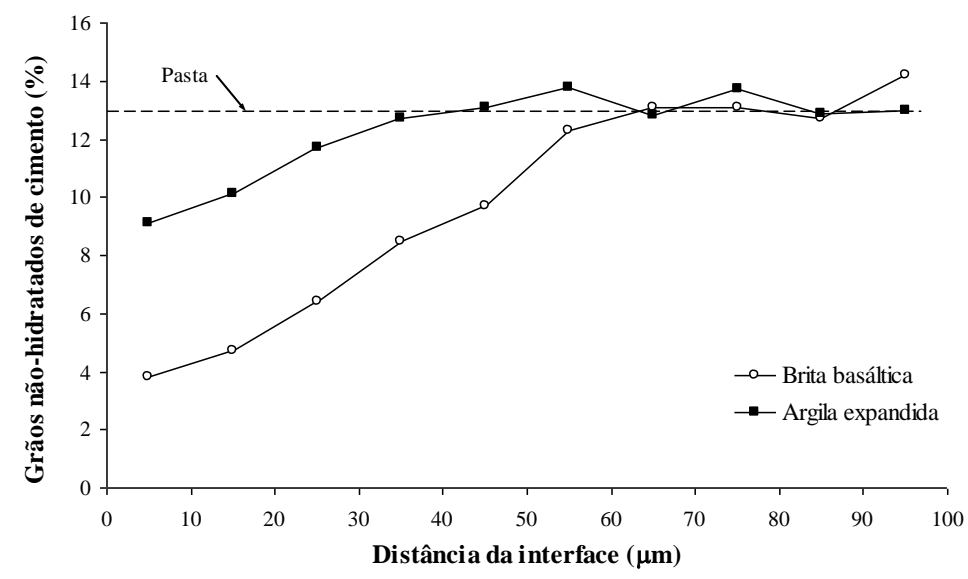

Figura 7 - Teor de grãos não hidratados de cimento Portland em função da distância da interface pasta/agregado

Observa-se, ainda, que a zona de transição dos concretos com argila expandida apresenta menor porosidade e maior teor de grãos não hidratados de cimento Portland, quando comparado ao concreto com brita basáltica.

Esses resultados reforçam a teoria de que a zona de transição em concreto com agregados leves com porosidade permeável na face externa, como a argila expandida brasileira, apresenta natureza distinta da observada nos concretos com agregados tradicionais. Nesses casos, a argila expandida promove a redução da espessura da zona de transição assim como da porosidade e do teor de hidróxido de cálcio $(\mathrm{CH})$, em função da diminuição da relação água/cimento da pasta nessa região pela absorção de água do agregado leve, efeito esse denominado de "filtragem" ou "densificação".

Esses resultados auxiliam no melhor entendimento da influência da argila expandida brasileira na microestrutura da zona de transição pasta/agregado em concretos leves estruturais e, por consequiência, nas propriedades desse material. Apesar de o concreto leve estrutural apresentar ampla utilização em função dos benefícios promovidos pela redução da massa específica, como a redução de esforços na estrutura, a economia com formas e cimbramento, bem como a diminuição dos custos com transporte e montagem de construções préfabricadas, a substituição dos agregados convencionais por agregados leves pode ocasionar alterações significativas em outras importantes propriedades do concreto, tais como resistência mecânica, módulo de deformação, estabilidade dimensional e isolamento térmico.

A redução da espessura e a melhoria da qualidade da zona de transição pasta/agregado promovida pela argila expandida, assim como a maior similaridade entre os valores do módulo de deformação do agregado leve e da argamassa, contribuem para a utilização mais eficiente da resistência mecânica potencial da argamassa em concretos com agregados leves e para o aumento do trecho linear da curva tensão/deformação, até cerca de $80 \%$ do carregamento último, enquanto nos concretos com agregados convencionais esse valor é de cerca de $60 \%$ (ROSSIGNOLO; AGNESINI, 2002, 2005).

Atribui-se, também, à melhoria da qualidade da zona de transição pasta/agregado a redução da permeabilidade dos concretos leves, em comparação aos concretos com agregados convencionais. Normalmente, a zona de transição pasta/agregado é a região de origem das primeiras microfissuras do concreto, quando solicitado mecanicamente, e apresenta maior permeabilidade do que a pasta, bem como altos teores de poros, de hidróxido de sódio e de etringita. Esse efeito pode ser observado no fenômeno da carbonatação, que ocorre mais intensamente em regiões com maior permeabilidade, permitindo a rápida difusão do dióxido de carbono, com maior quantidade de hidróxido de cálcio, como ocorre na zona de transição de concretos convencionais com elevada relação água/cimento (ROSSIGNOLO; AGNESINI, 2004, 2005).

\section{Conclusões}

Os resultados obtidos neste estudo indicaram que a zona de transição dos concretos com argila expandida apresenta menor espessura e menores porosidade e teor de hidróxido de cálcio do que o observado nos concretos com agregados convencionais, em função da diminuição da relação água/cimento da pasta nessa região, ocasionada pela absorção de água do agregado. 
Nos concretos com argila expandida, o valor obtido para a espessura da zona de transição foi de $30 \mu \mathrm{m}$, enquanto nos concretos com agregado basáltico foi de $55 \mu \mathrm{m}$.

\section{Referências}

CHEN, S. W.; SCHNEIDER, U. Microhardness and Mechanical Behavior of the Expanded Shale Concrete. In: KATZ A et al. (ed.) The Interfacial Transition Zone in Cementitius Composites. Londres: E\&FN SPON, 1998. p. 243-250.

ELSHARIEF, A.; COHEN, M. D.; OLEK, J. Influence of Lightweight Aggregate on the Microstructure and Durability of Mortar. Cement and Concrete Research, v. 35, n. 7, p. 1368-1376, 2005.

ELSHARIEF, A.; COHEN, M. D.; OLEK, J. Influence of Aggregate size, Water cement Ratio and Age on the Microstructure of the Interfacial Transition zone. Cement and Concrete Research, v. 33, n. 11, p.1837-1849, 2003.

HOLM, T. A.; BREMNER, T. W. State-of-theArt Report on High-Strength, High-Durability Structural Low-Density Concrete for Applications in Severe Marine Environments. US Army Corps of Engineers - Engineer Research and Development Center. ERDC/SL TR-00-3. 2000. 116 p.

KJELLSEN, K. O.; WALLEVIK, O. H.; FJÄLLBERG, L. Microstructures and Microchemistry of the Paste-Aggregate Interfacial Transition Zone of High-Performance Concrete. Advances in Cement Research, v. 10, n. 1, p. 3340, 1998.

LO, Y.; GAO, K. F.; JEARY, A. P. Microstructure of Pre-Wetted Aggregate on Lightweight Concrete. Building and Environment, v. 34, n. 6, p. 759 764, Nov. 1999.

MONTEIRO, P. J. M. Caracterização da Microestrutura do Concreto: fases e interfaces: aspectos de durabilidade e de microfissuração. 1993. 138 f. Tese (Livre-docência) - Escola Politécnica, Universidade de São Paulo, São Paulo, 1993.

PAULON, V. A. A Microestrutura do Concreto Convencional. In: ISAIA, G. C. (Org.). Concreto: ensino, pesquisa e realizações. São Paulo: Ibracon, 2005. v. 1, p. 583-604.
ROSSignolo, J. A. Concreto leve de Alto Desempenho Modificado com SB para PréFabricados Esbeltos: dosagem, produção, propriedades e microestrutura. 2003. $220 \mathrm{f}$. Tese (Doutorado em Ciências e Engenharia de Materiais) - Escola de Engenharia de São Carlos, Universidade de São Paulo, São Carlos, 2003.

ROSSIGNOLO, J. A.; AGNESINI, M. V. C. Concreto Leve Estrutural. In: ISAIA, G. C. (Org.). Concreto: ensino, pesquisa e realizações. São Paulo: Ibracon, 2005. v. 2, p. 1333-1362.

ROSSIGNOLO, J. A.; AGNESINI, M. V. C. Durability of Polymer-Modified Lightweight Aggregate Concrete. Cement and Concrete Composites, v. 26, n. 4, p. 375-380, 2004.

ROSSIGNOLO, J. A.; AGNESINI, M. V. C. Mechanical Properties of Polymer-Modified Lightweight Aggregate Concrete. Cement and Concrete Research, v. 32, n. 3, p. 329-334, 2002.

SARKAR, S. L.; CHANDRA, S.; BERNTSSON, L. Interdependence of Microstructure and Strength of Structural Lightweight Aggregate Concrete. Cement and Concrete Composites, v. 14, n. 4, p. 239-248, 1992.

TAYLOR, H. F. W.; NEWBURY, D. E. An Electron Microprobe Study of a Mature Cement Paste. Cement and Concrete Research, v. 14, n. 4, p. 565-573, 1984.

TEZUKA, Y. Concreto Leve à Base de Argila Expandida. 1973. Dissertação (Mestrado em engenharia Civil) - Escola Politécnica, Universidade de São Paulo, São Paulo, 1973.

VIEIRA, M. G. Betões de Elevado Desempenho com Agregados Leves: durabilidade e microestrutura. 2000. Dissertação (Mestrado em Engenharia Civil) - Instituto Superior Técnico, Universidade Técnica de Lisboa, Lisboa, 2000.

WASSERMAN, R.; BENTUR, A. Interaction between Fly Ash Aggregate With Cement Paste Matrix. In: KATZ, A. et al. (Ed.). The Interfacial Transition Zone in Cementitius Composites. Londres: E\&FN SPON, 1998. p. 267-275.

WASSERMAN, R.; BENTUR, A. Effect of Lightweight Fly Ash Aggregate Microstructure on the Strength of Concretes. Cement and Concrete Research, v. 27, n. 4, p. 525-537, 1997. 
WASSERMAN, R.; BENTUR, A. Interfacial interactions in lightweight aggregate concretes and their influence on the concrete strength. Cement and Concrete Composites, v. 18, p. 67-76, 1996.

ZHANG, M. H.; GJ $\phi R V$, O. E. Penetration of Cement Paste Into Lightweight Aggregate.

Cement and Concrete Research, v. 22, n. 1, p. 47-55, jan. 1992.
ZHANG, M. H.; GJ $\phi R V$, O. E. Microstructure of the Interfacial Zone Between Lightweight Concrete and Cement Paste. Cement and Concrete Research, v. 20, n. 4, p. 610-618, jul. 1990.

\section{Agradecimentos}

O autor agradece ao Laboratório Nacional de Engenharia Civil (LNEC) de Lisboa, Portugal, o apoio na realização das análises experimentais. 